\title{
Spin-liquid phase in an anisotropic triangular-lattice Heisenberg model: Exact diagonalization and density-matrix renormalization group calculations
}

\author{
M. Q. Weng, ${ }^{1}$ D. N. Sheng, ${ }^{1}$ Z. Y. Weng, ${ }^{2}$ and Robert J. Bursill ${ }^{3}$ \\ ${ }^{1}$ Department of Physics and Astronomy, California State University, Northridge, California 91330, USA \\ ${ }^{2}$ Center for Advanced Study, Tsinghua University, Beijing 100084, China \\ ${ }^{3}$ School of Physics, University of New South Wales, Sydney, New South Wales 2052, Australia
}

(Received 13 June 2006; published 24 July 2006)

\begin{abstract}
Based on exact diagonalization and density matrix renormalization group method, we show that an anisotropic triangular lattice Heisenberg spin model has three distinct quantum phases. In particular, a spin-liquid phase is present in the weak interchain coupling regime, which is characterized by an anisotropic spin structure factor with an exponential-decay spin correlator along the weaker coupling direction, consistent with the $\mathrm{Cs}_{2} \mathrm{CuCl}_{4}$ compounds. In the obtained phase diagram, the spin-liquid phase is found to persist up to a relatively large critical anisotropic coupling ratio $J^{\prime} / J=0.78$, which is stabilized by strong quantum fluctuations, with a parity symmetry distinct from two magnetic ordered states in the stronger coupling regime.
\end{abstract}

DOI: 10.1103/PhysRevB.74.012407

PACS number(s): 75.10.Jm, 75.30.Ds, 71.10.Hf, 71.27.+a

Two-dimensional (2D) frustrated spin systems have attracted intensive studies as they may exhibit unconventional magnetic properties. ${ }^{1-4}$ The isotropic spin-1/2 Heisenberg antiferromagnet (HAFM) on a triangular lattice was a candidate for the realization of a disordered spin-liquid phase, ${ }^{1}$ but it turns out to exhibit a three-sublattice antiferromagneticlong-range-order (AFLRO) as established by analytic ${ }^{5-8}$ and numerical ${ }^{5,9,10}$ studies. Among various spin models, a spin-liquid phase has been established for more geometrically frustrated systems on the Kagome lattice, ${ }^{11,12}$ dimer models, ${ }^{13}$ and models involving four spin exchange terms. ${ }^{14}$ The Heisenberg models on the square lattice with thirdnearest-neighbor couplings may also have a spin-liquid ground state as revealed by recent numerical studies based on density matrix renormalization group (DMRG) calculations. ${ }^{15}$

From the experimental point of view, the HAFM on an anisotropic triangular lattice is particularly interesting as it is directly relevant to the quantum magnet in the $\mathrm{Cs}_{2} \mathrm{CuCl}_{4}$ compounds, ${ }^{16-18}$ which may be described by a minimal model at half-filling (Ref. 19):

$$
H=J \sum_{\langle i, j\rangle} \mathbf{S}_{i} \cdot \mathbf{S}_{j}+J^{\prime} \sum_{\langle\langle i, j\rangle\rangle} \mathbf{S}_{i} \cdot \mathbf{S}_{j}
$$

Here $\mathbf{S}_{i}$ are spin-1/2 operators, and $J, J^{\prime} \geqslant 0$ are the nearestneighbor couplings along the chain $(J)$ and the other two axes $\left(J^{\prime}\right)$ between different chains on a triangular lattice.

Based on the variational Monte Carlo (VMC) method, a resonating valence bond (RVB) wave function was previously proposed ${ }^{19}$ to describe the low-lying anisotropic spin excitation observed experimentally ${ }^{16-18}$ in these systems, which suggests a gapless spin-liquid state. The model has also been studied by different analytic approaches such as spin wave theory (SWT), ${ }^{6}$ large- $S$ expansion, ${ }^{8}$ as well as the series expansion. ${ }^{20,21}$ These works have predicted magnetic ordered states at $J^{\prime} \geqslant 0.3 J \pm 0.03 J$ side, while the magnetic order vanishes on the smaller $J^{\prime}$ side suggesting a disordered phase. The recent series expansion study by Zheng et al. ${ }^{21}$ has further indicated that quantum renormalizations strongly enhance the one dimensionality of the spectra, which implies that a more accurate description of quantum effects is needed. Thus exact calculations with taking into account all the quantum fluctuations are highly desirable in order to further establish of existence of the spin-liquid phase, properly determine the quantum phase diagram as well as the nature of quantum phase transitions.

In this paper, we present a systematic numerical study of the magnetic phase diagram of the HAFM model in the spatially anisotropic triangular lattice at zero temperature by using exact diagonalization (ED) and DMRG methods. The main results are shown in Fig. 1, where three quantum phases are found with very distinctive magnetic structure factors. At small $J^{\prime} / J$, the ground state is a disordered spinliquid state, which smoothly connects to one-dimensional (1D) decoupled spin chains in the limit of $J^{\prime} \rightarrow 0$. The long range three-sublattice spiral Néel ordered phase occurs at an intermediate $J^{\prime} / J \sim 1$, and then a collinear Néel ordered phase appears beyond a larger $J^{\prime} / J$. In particular, we find that the regime of the spin-liquid phase extends over to a critical value, $J_{c 1}^{\prime} / J=0.78 \pm 0.05$, which is significantly larger than the one for magnetic disorder phase determined by pre-
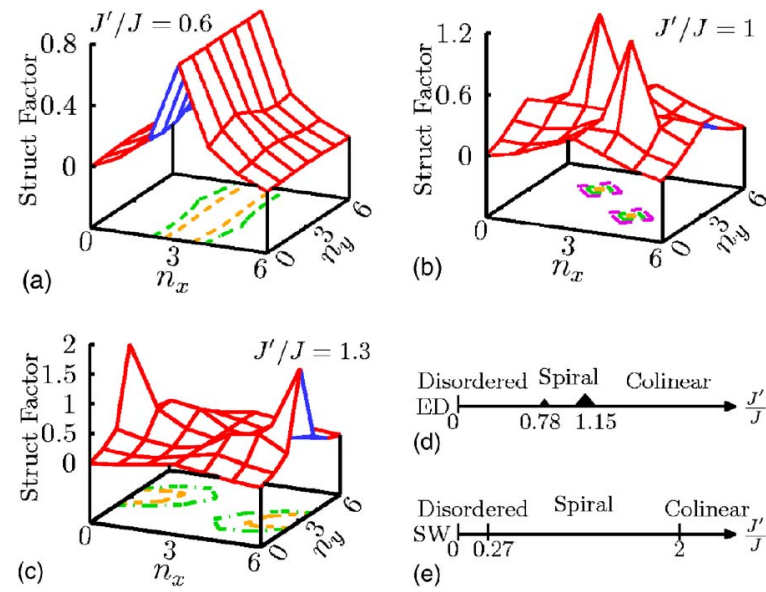

(e)

FIG. 1. (Color online) The static structure factor for $N=6 \times 6$ site at different coupling strengths: (a) $J^{\prime} / J=0.6$; (b) $J^{\prime} / J=1$; (c) $J^{\prime} / J=1.3$. (d) and (e) are the phase diagrams obtained from the ED in the present work and the linear SWT (Ref. 6), respectively. 
vious theoretical approaches. ${ }^{6,8,20}$ Such a phase boundary has been reliably identified by a vanishing first excitation energy, coinciding with the change of the ground state symmetry. By further performing a DMRG calculation ${ }^{22-24}$ with periodic boundary condition (PBC), ${ }^{25}$ we are able to establish an exponential decay of the equal-time spin correlation function between different chains, a hallmark for the 2D spin-liquid phase. ${ }^{26}$

Specifically, we consider a finite size system on the torus with length vectors $\mathbf{L}_{1}=N_{1} \mathbf{a}_{1}$ and $\mathbf{L}_{2}=N_{2} \mathbf{a}_{2}$ connecting identical sites (i.e., a PBC system). Here $\mathbf{a}_{1}$ and $\mathbf{a}_{2}\left[\mathbf{a}_{1}=(1,0)\right.$, $\mathbf{a}_{2}=(1 / 2, \sqrt{3} / 2)$ in real space] are two primitive vectors of the triangular lattice. The coupling strength along $\mathbf{a}_{1}$ is $J$ and along both $\mathbf{a}_{2}$ and $\mathbf{a}_{3}=\mathbf{a}_{2}-\mathbf{a}_{1}$ is $J^{\prime}$. The total number of sites is $N=N_{1} \times N_{2}$. The ground state is determined by a Lanczos diagonalization of the Hamiltonian using all symmetries ${ }^{9,27}$ for system sizes up to $N=36$ (corresponding to a Hilbert space of a dimension $N_{H}=63092837$ ). On the other hand, the DMRG method ${ }^{23,24}$ allows us to extend the exact calculation to larger systems up to $N=8 \times 18$ sites (i.e., 8 -legs). ${ }^{25}$

We first present the structure factor $(\mathrm{SF})$ of the statistic spin correlation $S(\mathbf{Q})=\sum_{i j} e^{i \mathbf{Q} \cdot\left(\mathbf{R}_{i}-\mathbf{R}_{j}\right)}\left\langle S_{j}^{z} S_{i}^{z}\right\rangle / N$ in Fig. 1 for a system of $6 \times 6$ sites at $J^{\prime}=0.6 J, J^{\prime}=J$, and $J^{\prime}=1.3 J$, respectively. For the smallest $J^{\prime}$ [shown in Fig. 1(a)], $S(\mathbf{Q})$ is peaked along the lines $n_{x}= \pm 3$, corresponding to magnetic wave vectors $Q_{x}= \pm \pi$, while it only shows a slight dependence on $n_{y}$ [the components of $\mathbf{n}$ used here are related to the wave vectors $Q$ by $Q_{x}=2 \pi n_{x} / N_{1}$ and $Q_{y}=4 \pi\left(n_{x} / 2 N_{1}\right.$ $\left.\left.+n_{y} / N_{2}\right) / \sqrt{3}\right]$. This is a typical feature of weakly coupled spin chains with strong antiferromagnetic spin correlations within each chain, whereas the correlations between the chains are short ranged (see also Fig. 3 below). As $J^{\prime}$ is increased towards the isotropic point $J^{\prime}=J$ [shown in Fig. $1(\mathrm{~b})$ ], $S(\mathbf{Q})$ is qualitatively changed with the peaks moving to $\mathbf{n}= \pm(4,-2)$, corresponding to wave vectors $\mathbf{Q}$ $= \pm(4 \pi / 3,0)$ which represents the three-sublattice (spiral) Néel state, in agreement with the AFLRO ground state for the isotropic triangular lattice. ${ }^{8,10,27}$ In the region of 0.78 $<J^{\prime}<1.15$, besides the major peaks at $\mathbf{n}= \pm(4,-2)$, there are also minor peaks at $\mathbf{n}=(0, \pm 3)$ as well, which may indicate the incommensurate spin correlation in this region. When $J^{\prime}$ is further increased to $J^{\prime} / J=1.3$ [shown in Fig. $1(\mathrm{c})]$, the peaks of $S(\mathbf{Q})$ move to $\mathbf{n}=(0, \pm 3)$ or $\mathbf{Q}$ $=(0, \pm 2 \pi / \sqrt{3})$, which are exactly the ordering vectors for a long-range collinear Néel order along both $\mathbf{a}_{2}$ and $\mathbf{a}_{3}$ axes.

Thus we have seen that when the anisotropy parameter $J^{\prime} / J$ varies from 0 to 2 , the ground states exhibit three distinct phases with the SF structures dramatically different from each other. The corresponding phase diagram is given in Fig. 1(d), where two critical points $J_{c 1}^{\prime} / J=0.78 \pm 0.05$ and $J_{c 2}^{\prime} / J=1.15 \pm 0.1$ separate the spin disordered phase from the left side (the small $J^{\prime}$ regime), the spiral Néel ordered phase in the middle, and the collinear Néel ordered phase on the right side (the large $J^{\prime}$ regime), respectively.

As $J^{\prime} / J$ crosses the phase boundaries in Fig. 1(d), not only the characteristic SF changes, but the low energy spectrum also qualitatively changes. In Fig. 2, $\Delta E$ as the energy difference (per site) between the first excited state $E_{1}$ and the ground state $E_{0}$ is plotted as a function of $J^{\prime} / J$ for three

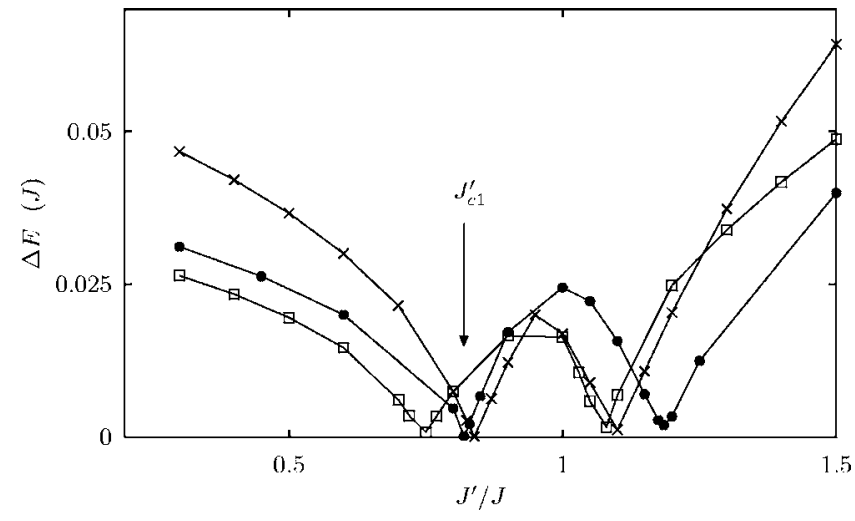

FIG. 2. The excitation energy $\Delta E=\left(E_{1}-E_{0}\right) / N$, for system sizes $6 \times 6(\bullet), 8 \times 4(\square)$, and $6 \times 4(\times)$, in units of $J$.

different system sizes: $N=6 \times 4,8 \times 4$, and $6 \times 6$. As $J^{\prime} / J$ is increased from the weak side, $\Delta E$ decreases monotonically. Remarkably, at the critical $J_{c 1}^{\prime}, \Delta E$ vanishes exactly where the ground state and the first excited state crosses each other. As a matter of a fact, the parity symmetry (the reflection along a symmetric axis of the systems) for the ground state also undergoes a change, from the odd parity at $J^{\prime}<J_{c 1}^{\prime}$ to the even one at $J^{\prime}>J_{c 1}^{\prime}$. The vanishing $\Delta E$ at finite $N$ 's and a discrete symmetry change suggest a first-order phase transition occurring at $J_{c 1}^{\prime}$. Here it is noted that the qualitative behavior of the SF as a function of $J^{\prime}$ is independent of the system sizes in the ED calculation (with $N=24-36$ ) and the phase boundary for the spin liquid phase shown in Fig. 2 do not change much when $N$ is changed, both suggesting a weak finite-size effect in our calculation. When $J^{\prime} / J$ is further increased to around $J_{c 2}^{\prime}=1.15 \pm 0.1, \Delta E$ reaches to an another minimum with a finite but very small value $[\Delta E$ $\simeq(0.0015 \pm 0.0005) J]$. Together with the observation in a drastic change in the SF around this point, it indicates that the system undergoes another phase transition from the spiral Néel state to the collinear Néel ordered phase. It could be a continuous transition, and $\Delta E$ is expected to vanish in the thermodynamic limit $N \rightarrow \infty$.

One of the most interesting features of the phase diagram determined by the ED in Fig. 1(d) is that the regime of the spin-liquid phase on the small $J^{\prime}$ side persists up to $J_{c 1}^{\prime}$ $\sim 0.78 \mathrm{~J}$, which is much larger than $\sim 0.3 \mathrm{~J}$ obtained by the linear SWT (Ref. 6) shown in Fig. 1(e). This difference may be attributed to the importance of quantum fluctuations ${ }^{21}$ in the model, which could have been underestimated in the linear SWT approach. We have carried out a standard SWT calculation by taking into account the magnon scattering and the geometrical frustration ${ }^{7,9}$ up to the second order. Our result shows that the boundary of the disordered region indeed moves up to $J_{c 1}^{\prime}=0.39 \mathrm{~J}$ from the linear SWT $J_{c 1}^{\prime}$ $=0.27 J$. The upper boundary of the spiral ordered state is around $J^{\prime} / J \sim 1.15$, which is close to the one obtained by series expansion in Ref. 20, while both series expansion and spin wave theory suggest that spin disordered state may survive in a region or at a point between spiral state and colinear state. ${ }^{6}$ However, we are not able to directly check this conjecture due to the limited system size, although the fast change of SF within a small region $1.15 \leqslant J^{\prime} / J \leqslant 1.2 \mathrm{might}$ indicate the existence of a disordered state. ${ }^{20}$ 
To check the possibility of realizing an incommensurate spiral ordered state disfavored by PBC in a small system, we carry out the calculation with a twisted boundary condition (TBC) $\mathbf{S}_{\mathbf{r}_{i}+\mathbf{L}_{\alpha}}=R_{\mathbf{z}}\left(\mathbf{Q} \cdot \mathbf{L}_{\alpha}\right) \mathbf{S}_{\mathbf{r}_{i}}{ }^{11,27}(\alpha=1,2)$, with $\mathbf{Q}$ being the classic magnetic wave vector and $R_{\mathbf{z}}$ being the spin-rotation operator. It is found that in the region of $J_{c 1}^{\prime}<J^{\prime}<J_{c 2}^{\prime}$, the ground state energy of $E_{0}$ with TBC is slightly lower than the one with $\mathrm{PBC}$ (for 24-36 sites systems). Once $J^{\prime}$ goes beyond this region, $\mathrm{PBC}$ always gives smaller ground state energy. Moreover, in the spiral ordered region $\left(J_{c 1}^{\prime}, J_{c 2}^{\prime}\right)$, the ground state energy from the ED method is close to that obtained by the series expansion $E_{0}(S E),{ }^{20}$ which may be numerically accurate if suitable expansion states are chosen. However, outside this region, $E_{0}$ from ED is systematically lower than the energy $E_{0}(S E)$ with spiral order, which indicates that the spiral ordered state may not be stable outside the region of $\left(J_{c 1}^{\prime}, J_{c 2}^{\prime}\right)$.

To further examine the magnetic characterization of the spin-liquid phase, we have also performed the DMRG calculation for larger system sizes. In particular, our DMRG code works with PBC (Ref. 24), which can reproduce all the ED results for $N \leqslant 36$ systems and extend the study up to a system size of $8 \times 18$. The system is setup as four blocks with replacing the single-site block in standard DMRG by a block of $N_{1} / 2$ sites. ${ }^{25}$ In the insets of Fig. 3, we present the relative error of the ground state energy $\Delta=\frac{E_{0}(m)-E_{0}}{E_{0}}$ as a function of state number $m$ kept in the main block in the DMRG calculation for $6 \times 6$ system. Here $E_{0}$ are the ground state energy from the ED calculation. By keeping up to $m=500$, we obtain an accuracy lower than $\Delta=1.4 \times 10^{-5}$ for the PBC system. For a longer length system, by extrapolating the $E_{0}(m)$ to large $m$ limit to obtain an estimate of the ground state energy to serve as $E_{0}$ (where no exact results exist), we found that the error bar remains around $10^{-5}$ for all six-leg systems and around $10^{-4}$ for eight-leg systems as long as we keep $m$ up to 800 with a discarded density matrix weight around $10^{-6}$. We have also applied the standard DMRG method $^{23}$ by keeping $m=1500$ states (with adding a single site to each block) and the same ground state energies with a similar error bar were obtained for both six-leg and eight-leg systems.

In Fig. 3, we present the spin correlation function $C(y)$ $=\left\langle S_{0}^{z} S_{y \mathbf{a}_{2}}^{z}\right\rangle$ as a function of the distance between two sites along the $\mathbf{a}_{2}$ axis (weaker coupling direction). In Fig. 3(a) for $J^{\prime}=0.6 J$, one first notices that the data from the ED on a 6 $\times 6$ lattice agrees quite well with the DMRG results for larger system sizes $(N=6 \times 18$ and $8 \times 18)$. $|C(y)|$ decreases by about six orders of magnitude as $y$ increases, following an exponential-decay law which can be well fitted by the dashed line: $|C(y)| \propto e^{-y / 0.544}$ at $y<L_{2} / 2$. As our system is on a torus (PBC), $|C(y)|$ turns back when $y$ exceeds the half of $L_{2}$. In Fig. 3(b), $|C(y)|$ at a weaker $J^{\prime}=0.3 J$ is shown, which exhibits similar behavior, except that the exponential-decay correlation length has been reduced from 0.544 to 0.418 . On the other hand, along the chain direction $\left(\mathbf{a}_{1}\right)$, we have found that $C(x)$ follows a power-law behavior at small $J^{\prime}$, consistent with the one-dimensional peaks in the SF shown in Fig. 1(a). The remarkable similarity in the spin correlator be-
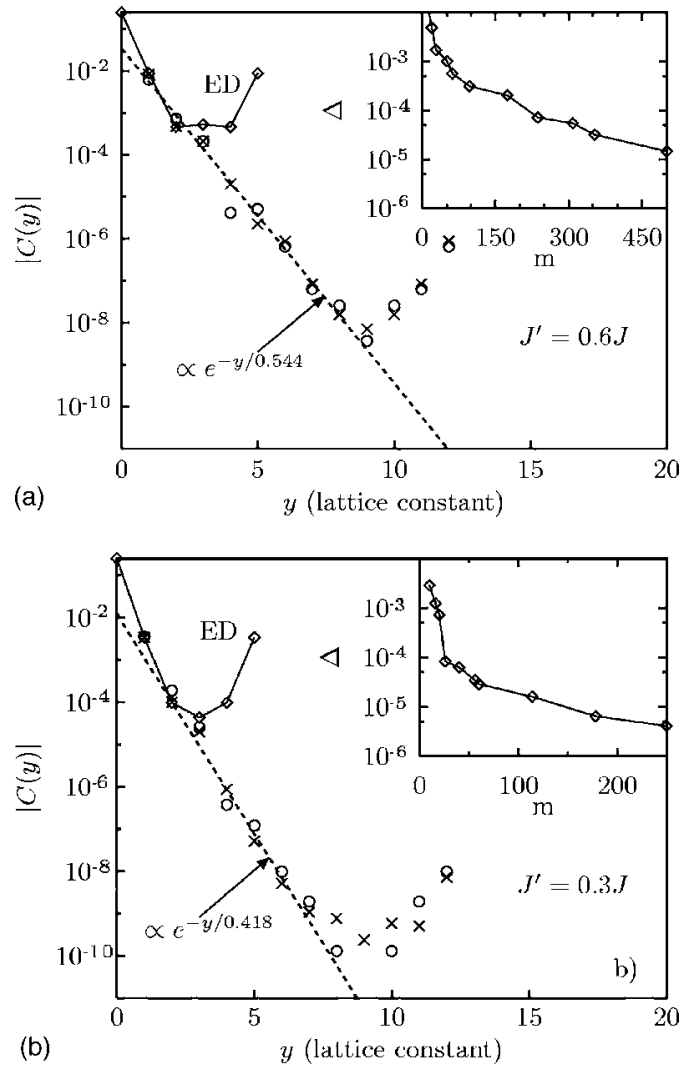

FIG. 3. Absolute value of spin-spin correlation function $|C(y)|$ $=\left|\left\langle S_{0}^{z} S_{y \mathbf{a}_{2}}^{z}\right\rangle\right|$ along the $\mathbf{a}_{2}$ direction in six-leg system $(\times)$ with $N=6$ $\times 18$ and eight-leg system $(\bigcirc)$ with $N=8 \times 18$ at different coupling strengths: (a) $J^{\prime} / J=0.6$; (b) $J^{\prime} / J=0.3$. We also present the corresponding $|C(y)|$ from the ED at $N=6 \times 6$ for comparison. The dashed lines are the fittings to the corresponding data by exponential functions. The insets are accuracy of the ground state energy $\Delta=\left|\left(E(m)-E_{0}\right) / E_{0}\right|\left(E_{0}\right.$ is the exact energy from ED) as a function of state number $m$ kept in each block in the DMRG calculation for $6 \times 6$ system.

tween $J^{\prime}=0.6 J$ and $J^{\prime}=0.3 J$ further supports the ED phase diagram where spin-liquid phase is extended to a large critical $J_{c 1}^{\prime}=(0.78 \pm 0.05) J$. However, we point out that we cannot exclude the possibility of the system developing an extremely small magnetic order or breaking lattice symmetry in the thermodynamic limit, which may not be revealed by finite-size calculations.

In conclusion, we have performed the ED and DMRG calculations of the HAFM on an anisotropic triangular lattice. We have demonstrated that the ground states of this system vary from the disordered spin-liquid state to a spiral ordered state, and then to a collinear Néel ordered state, when the anisotropic parameter $J^{\prime} / J$ is continuously increased. From the characteristic features of the spin structure factor, the first excitation energy, as well as the exponential decay behavior of the spin correlator, we establish the existence and robustness of a spin-liquid phase, beyond a previous VMC study. ${ }^{19}$ The characterization of such a spin-liquid phase demands further theoretical studies. ${ }^{28}$ Our results provide a possible explanation of the spin-liquid behavior in the dynamic structure factor and the quasi-1D spin excitation spectrum found in the $\mathrm{Cs}_{2} \mathrm{CuCl}_{4}$ compounds. Stabilized by 
quantum fluctuations, such a spin liquid phase is also expected to be observed experimentally in systems with much stronger interchain couplings.

D.N.S. would like to thank L. Balents for stimulating discussions. The authors would like to thank W. Zheng for providing us the ground state energy of series expansions. This work is supported by ACS-PRF 41752-AC10, Research Corporation Grant No. CC5643, NSF Grants Nos. DMR-0307170 and DMR-0605696 (MQW, DNS), and NSFC Grants Nos. 10374058 and 90403016 (ZYW).
${ }^{1}$ P. Fazekas and P. W. Anderson, Philos. Mag. 30, 423 (1974).

${ }^{2}$ G. Baskaran and P. W. Anderson, Phys. Rev. B 37, 580 (1988).

${ }^{3}$ S. A. Kivelson, D. S. Rokhsar, and J. P. Sethna, Phys. Rev. B 35, 8865 (1987).

${ }^{4}$ X. G. Wen, Phys. Rev. B 44, 2664 (1999); T. Senthil and M. P. A. Fisher, ibid. 63, 134521 (2001); Phys. Rev. Lett. 86, 292 (2001).

${ }^{5}$ Quantum Magnetism, edited by U. Schollwock, J. Richter, D. Farnell, and R. Bishop (Springer, Berlin, 2004).

${ }^{6}$ A. E. Trumper, Phys. Rev. B 60, 2987 (1999); J. Merino, R. H. McKenzie, J. B. Marston, and C. H. Chung, J. Phys.: Condens. Matter 11, 2965 (1999); A. E. Trumper, L. Capriotti, and S. Sorella, Phys. Rev. B 61, 11529 (2000).

${ }^{7}$ A. V. Chubukov, S. Sachdev, and T. Senthil, J. Phys.: Condens. Matter 6, 8891 (1994).

${ }^{8}$ C. H. Chung, J. B. Marston, and R. H. McKenzie, J. Phys.: Condens. Matter 13, 5159 (2001).

${ }^{9}$ P. W. Leung and K. J. Runge, Phys. Rev. B 47, 5861 (1993).

${ }^{10}$ L. Capriotti, A. E. Trumper, and S. Sorella, Phys. Rev. Lett. 82, 3899 (1999).

${ }^{11}$ P. Lecheminant, B. Bernu, C. Lhuillier, L. Pierre, and P. Sindzingre, Phys. Rev. B 56, 2521 (1997).

${ }^{12}$ L. Balents, M. P. A. Fisher, and S. M. Girvin, Phys. Rev. B 65 , 224412 (2002); D. N. Sheng and L. Balents, Phys. Rev. Lett. 94, 146805 (2005).

${ }^{13}$ D. S. Rokhsar and S. A. Kivelson, Phys. Rev. Lett. 61, 2376 (1988); R. Moessner and S. Sondhi, ibid. 86, 1881 (2001).

${ }^{14}$ G. Misguich, B. Bernu, C. Lhuillier, and C. Waldtmann, Phys. Rev. Lett. 81, 1098 (1998).

${ }^{15}$ L. Capriotti, D. J. Scalapino, and S. R. White, Phys. Rev. Lett.
93, 177004 (2004).

${ }^{16}$ R. Coldea, kD. A. Tennant, A. M. Tsvelik, and Z. Tylczynski, Phys. Rev. Lett. 86, 1335 (2001).

${ }^{17}$ R. Coldea, D. A. Tennant, K. Habicht, P. Smeibidl, C. Wolters, and Z. Tylczynski, Phys. Rev. Lett. 88, 137203 (2002).

${ }^{18}$ R. Coldea, D. A. Tennant, and Z. Tylczynski, Phys. Rev. B 68, 134424 (2003).

${ }^{19}$ S. Yunoki and S. Sorella, Phys. Rev. Lett. 92, 157003 (2004).

${ }^{20}$ W. Zheng, R. H. McKenzie, and R. P. Singh, Phys. Rev. B 59, 14367 (1999).

${ }^{21}$ W. Zheng, J. O. Fjaerestad, R. P. Singh, R. H. McKenzie, and R. Coldea, Phys. Rev. Lett. 96, 057201 (2006).

${ }^{22}$ S. R. White, Phys. Rev. B 48, 10345 (1993).

${ }^{23}$ S. R. White and D. J. Scalapino, Phys. Rev. Lett. 80, 1272 (1998).

${ }^{24}$ R. J. Bursill, Phys. Rev. B 60, 1643 (1999).

${ }^{25}$ This new 2D DMRG code uses four blocks, each of the middle two blocks have half of sites from one-leg (for eight-leg system with $N_{1}=8$, there are $2^{N_{1} / 2}=16$ states in this block), instead of the more conventional one-site structure. Thus the $2 \mathrm{D}$ structure as well as the PBC can be well taken into account ${ }^{24}$ at the price of keeping a lot more states in the four-block system. We keep up to $m=800$ state in the big-block and diagonalize four-block system with about one third of $800 \times 800 \times 2^{N_{1}} \sim 50 \times 10^{6}$ (for eight-legs) states using total $S^{z}$ as a good quantum number.

${ }^{26}$ T. Senthil and M. P. A. Fisher, Phys. Rev. B 62, 7850 (2000).

${ }^{27}$ B. Bernu, P. Lecheminant, C. Lhuillier, and L. Pierre, Phys. Rev. B 50, 10048 (1994).

${ }^{28}$ J. Alicea, O. I. Motrunich, and M. P. A. Fisher, Phys. Rev. Lett. 95, 247203 (2005). 